\title{
The Influence of a Continuing Medical Education Campaign on New Strategies to Improve Appropriate Use of Antibiotics
}

\author{
Brenda A. Bucklin, ${ }^{1}$ Ronald S. Gibbs, ${ }^{1}$ Carolyn Wieber, ${ }^{1}$ and Leslie Myers ${ }^{2}$ \\ ${ }^{1}$ Office of Continuing Medical Education and Professional Development, University of Colorado School of Medicine, \\ Aurora, CO 80045, USA \\ ${ }^{2}$ Department of Obstetrics and Gynecology, University of Colorado School of Medicine, Aurora, CO 80045, USA
}

Correspondence should be addressed to Brenda A. Bucklin; brenda.bucklin@ucdenver.edu

Received 25 April 2015; Accepted 3 November 2015

Academic Editor: Friedrich Paulsen

\begin{abstract}
Copyright (C) 2015 Brenda A. Bucklin et al. This is an open access article distributed under the Creative Commons Attribution License, which permits unrestricted use, distribution, and reproduction in any medium, provided the original work is properly cited.

Introduction. Widespread use of antibiotics has led to drug-resistant bacteria and reports of drug-resistant infections. A continuing medical education (CME) campaign was used to improve antibiotic use among primary care providers. Methods. The Office of CME and Professional Development at the University of Colorado School of Medicine produces a semiannual, week-long course for primary care providers. A 2-year multifaceted CME campaign consisted of course content on antibiotic use, a practice audit, and two surveys to measure perceptions of the problem of antibiotic overuse, potential barriers to achieving appropriate use, and strategies to overcome barriers. Results. The overall response rate in the 2 nd part of the campaign was $68.4 \%$. Sixty-six percent of respondents had implemented at least one strategy to reduce antibiotic overuse. The rate was significantly higher among those who had attended previous reviews $(81.0 \%)$ compared with those who had attended neither $(54 \%, p=0.0002)$. However, there was no "dose effect" on the rate of implementing a new strategy. Conclusions. Overuse of antibiotic therapy has important public health implications. Results suggest that mixed interactive and didactic CME program was effective in increasing awareness of antibiotic overuse and strategies for reducing antibiotic administration.
\end{abstract}

\section{Introduction}

Continuing medical education (CME) plays a critical role in improving patient outcomes, enhancing the overall quality and efficiency of the healthcare system, and reducing overall healthcare costs. Although CME providers are required to comply with accreditation criteria from the Accreditation Council for Continuing Medical Education to (1) "integrate CME into the process for improving professional practice" (accreditation criterion 16); (2) "identify factors outside the provider's control that impact outcomes" (accreditation criterion 18); (3) and "implement educational strategies to remove, overcome, or address barriers to physician change" (accreditation criterion 19) [1], most CME activities are not designed to change behaviors in practice but focus on knowledge that reinforces quality of a physician's practice [2-4]. Recently, Légaré et al. [4] evaluated the objectives of 110 accredited continuing professional development activities to determine whether cognitive, affective, or psychomotor skills were targeted in the course objectives [2]. Only $26 \%$ of the activities were aimed at changing behavior by improving skills in analysis, synthesis, and evaluation while more than half of the activities were focused on knowledge and comprehension.

Although drug-resistant bacteria are emerging because of the widespread use of antibiotics both inside and outside medicine [5-7], antibiotic overuse in the United States is an important area of practice needing improvement. Reports suggest that up to $48 \%$ of patients with rhinopharyngitis and up to $73 \%$ of patients with acute bronchitis receive antibiotics even when these infections are unresponsive to antibiotic therapy [8-10]. There are increasing reports of drug-resistant infections and calls for judicious use of antibiotics in healthcare to slow the emergence of resistant organisms and to extend the use of current antibiotics [11]. Despite these initiatives, inappropriate use of antibiotics remains common. 
Recently Gibbs et al. [12] used this important area of clinical practice to foster and develop a change in behavior and improve the use of antibiotics in primary care. The first phase of the multifaceted two-part campaign was conducted to achieve appropriate use. Results of the survey indicated that $90 \%$ of learners perceived inappropriate antibiotic use as a "very big" or "big" problem in the United States, but only $44 \%$ perceived this about their practice. Learners identified multiple pressures (e.g., patient expectations, breaking old habits, and fear that patients would go elsewhere) to prescribe antibiotics. In this second part of the interactive campaign to educate clinicians regarding appropriate indications for antibiotic use, we report the results of CME course survey that determined if participants had made changes in practice based on attendance of the course.

\section{Materials and Methods}

This research qualified as exempt research after review by the Colorado Multi-Institutional Review Board.

For over 60 years, the Office of Continuing Medical Education and Professional Development at the University of Colorado School of Medicine has produced a semiannual, week-long course for primary care providers. The national audience is made up of approximately 65\% physicians (nearly all family physicians) and 35\% physicians' assistants or nurse practitioners. Many learners attend year after year.

As previously described [12], we began a continuing education campaign to improve appropriate use of antibiotics in 2012. The campaign consisted of course content on antibiotic use (12.5\% of course hours in 2012 and $17.1 \%$ in 2013 ), a sample practice audit in 2013, and two surveys. The first survey conducted at the 2012 course dealt with perceptions of the importance of the problem of inappropriate antibiotic use, perceived potential barriers to achieving appropriate use, and strategies that could reasonably be used to overcome these barriers. The second survey conducted in 2013 used characteristics that would likely lead the practitioner to prescribe an antibiotic for a patient with symptoms of acute bronchitis, a condition for which the Centers for Disease Control (CDC) does not recommend antibiotics. In this survey, we also asked whether the respondents had implemented any new strategies in the last year to avoid inappropriate use of antibiotics.

At the 2014 course, we asked attendees to complete a five-item questionnaire. The first question was whether the respondent had an outpatient practice. If no, that was the end of the survey. The second item was whether the respondent had attended either the 2012 or the 2013 courses. Third, we asked whether the attendee had implemented any new strategies in the last two years to improve appropriate use of antibiotics. If no, this was the end of the survey. If yes, we asked that respondent to circle all strategies implemented from a menu of 10 items. (See Table 3.)

Finally, we asked attendees if they had implemented one or more strategies. They were also asked to indicate the reason(s) for making the change in practice, again from a menu of 7 choices. Among these were CME at Family Medicine Review (FMR) course and other CME. (See Table 4 for a full menu of items.)
TABLE 1: Attendance of survey responders at previous Family Medical Reviews (FMRs), by year $(N=175)$.

\begin{tabular}{lc}
\hline Year attended & $N(\%)$ \\
\hline 2012 & $24 / 175(13.7)$ \\
2013 & $11 / 175(6.3)$ \\
Both & $44 / 175(25.1)$ \\
Neither & $96 / 175(54.9)$ \\
\hline
\end{tabular}

TABLE 2: Number of survey responders who implemented any new strategy in the last two years to improve appropriate antibiotic use, by attendance at previous Family Medical Review (FMR).

\begin{tabular}{lc}
\hline Previous FMR attendance & $\begin{array}{c}\text { Implemented any new strategy, } \\
N(\%)\end{array}$ \\
\hline Attended 2012 & $19 / 24(79.2)$ \\
Attended 2013 & $9 / 11(81.8)$ \\
Attended both & $36 / 44(81.8)$ \\
Attended neither & $52 / 96(54.2)$ \\
\hline
\end{tabular}

$p=0.0002$ comparing rate for those who attended one or both previous FMRs with rate for those who attended neither.

We tallied several key results, including number (\%) of respondents who had made changes in practice in the last two years and reasons for making the changes, and discussed the results on the last day of the conference in a programmatic evaluation. For formal analysis, data from paper forms was entered into a Redcap database and exported to SPSS for further statistical analysis. Standard descriptive statistics and Chi-square tests were employed to compare rates of discrete data, with $p$ values of less than 0.05 considered significant. Student's $t$-test was also used to determine if the number of strategies identified and reason(s) for implementing new strategies was different between attendees and nonattendees, again with $p$ values of less than 0.05 considered significant.

\section{Results}

The overall response rate in the 2014 survey was $68.4 \%$. We limited the rest of the survey to those who had an outpatient practice $(N=175)$. In Table 1 , we show the attendance of survey responders at FMR by year. Just under half had attended one or both of the two previous courses.

In Table 2, we show the number (\%) of responders who had implemented any new strategies to improve appropriate antibiotic use in the last two years.

Overall, $66.3 \%$ (116 of 175) had implemented at least one strategy, but we found that the rate was significantly higher among those who had attended one or both previous reviews (81.0\% [64 of 79]) compared with the rate for those who had attended neither (54.2\% [52 of 96], $p=0.0002)$. There was no "dose effect" in that the rate of implementing a new strategy was similar among those who attended 2012 only versus 2013 only versus both $(79.2 \%, 81.8 \%$, and $81.8 \%$, resp.). In Table 3 , we show the distribution of implemented strategies for both of those who had attended versus not attended the previous FMRs. 
TABLE 3: Identified new strategies implemented, by previous Family Medical Review (FMR) attendance.

\begin{tabular}{lcc}
\hline Strategy & \multicolumn{2}{c}{ Previous FMR attendance $(N, \%)$} \\
Never attended $(N=52)^{*}$ \\
\hline Patient education/educational & Attended $(N=64)^{*}$ & $32 / 52(61.6)$ \\
materials/informational sheets & $44 / 64(68.8)$ & $4 / 52(7.7)$ \\
Having paper copies of guidelines available & $13 / 64(20.3)$ & $15 / 52(28.8)$ \\
Accessing guidelines electronically & $18 / 64(28.1)$ & $7 / 52(13.5)$ \\
Developing a practice policy & $9 / 64(14.1)$ & $2 / 52(3.8)$ \\
Practice audit to see how my practice is doing & $9 / 64(14.1)$ & $12 / 52(23.1)$ \\
Signs in reception room/office & $18 / 64(28.1)$ & $23 / 52(44.2)$ \\
More physician education & $26 / 64(40.6)$ & $8 / 52(15.4)$ \\
Staff meetings to discuss this problem & $10 / 64(15.6)$ & $13 / 52(25.0)$ \\
More staff education & $14 / 64(21.9)$ & $4 / 52(7.7)$ \\
Others & $3 / 64(4.7)$ & \\
\hline
\end{tabular}

${ }^{*} \mathrm{~N}=$ number of survey respondents who implemented one or more new strategies.

TABLE 4: Identified reasons for making a change in protocol, by previous Family Medical Review (FMR).

\begin{tabular}{lcr}
\hline Reason for implementation & \multicolumn{2}{c}{ Previous FMR attendance, number $(\%)$} \\
Never attended $(N=52)^{*}$ \\
\hline CME at this course & Attended $(N=64)^{*}$ & $0 / 52(0.0)$ \\
Other CME I've taken & $56 / 64(87.5)$ & $22 / 52(42.3)$ \\
$\begin{array}{l}\text { Recommendations of national organizations } \\
\text { (such as CDC) }\end{array}$ & $41 / 64(20.3)$ & $38 / 52(73.1)$ \\
Medical articles I've read & $31 / 64(48.4)$ & $35 / 52(67.3)$ \\
$\begin{array}{l}\text { Discussions with practice partners or other } \\
\text { medical colleagues }\end{array}$ & $32 / 64(50.0)$ & $26 / 52(50.0)$ \\
$\begin{array}{l}\text { Policy from my medical employer, hospital or } \\
\text { medical group }\end{array}$ & $8 / 64(12.5)$ & $12 / 52(23.1)$ \\
Other & $4 / 64(6.3)$ & $1 / 52(1.9)$ \\
\hline
\end{tabular}

${ }^{*} N=$ number of survey respondents who had implemented one or more new strategies.

For both groups of survey responders, the most commonly implemented strategies were similar (namely, patient education, physician education, accessing electronic guidelines, and placing signs in reception room/office). Among previous attendees who reported implementing a new strategy, the average number of strategies implemented was 2.56 (164 among 64) whereas among those who had not attended but nevertheless reported a new strategy the average number of strategies implemented was 2.31 (120 among 52), $p=0.22$.

In the last question of the survey, we asked respondents to indicate the reason(s) for implementing new strategies. As shown in Table 4, among previous attendees, the leading reason was CME at previous FMRs.

However, we found that responders cited many reasons for implementing new strategies. The average number of reasons cited among previous attendees was 2.89 (185 among 64) whereas among those who had not attended but who reported a new strategy the average number of reasons cited was 2.58 (134 among 52), $p=0.15$.

\section{Discussion}

Antibiotic overuse has potential important health implications and serious adverse effects and creates unnecessary expense. Antibiotics are required to treat severe bacterial infections but antibiotic overuse has contributed to increased resistance. Bacterial resistance has been identified as an important world health threat [13] because even common bacteria can be transformed into multidrug-resistant bacteria or "superbugs." Overuse of antibiotics can fuel antibioticresistant bacteria and can lead to life-threating infections. To address the widespread problem, the CDC has created a campaign entitled "Get Smart" to educate providers and patients about appropriate prescribing and use of antibiotics [14]. Despite this campaign, a recent report by the CDC suggests that more than 2 million people in the United States become infected with drug-resistant bacteria each year and at least 23,000 people die from these infections annually [15].

Several studies demonstrate a benefit in participation in CME activities [3,16-20], but benefit is limited because most 
CME activities are didactic sessions and designed to address the lowest levels of cognitive learning (i.e., comprehend and apply knowledge) $[4,21]$. Our two-part study, using a multifaceted approach demonstrated that higher levels of cognitive learning were more likely to produce a behavior change in clinical practice. Previous course attendees were more likely to make a change in practice and implement at least one or more new strategies for appropriate use of antibiotics. However, for all responders who implemented a new strategy, the specific new strategies were similar and the number of new strategies implemented was similar whether or not the responders had attended a previous FMR course. We believe that the influence of having attended a previous FMR was not in developing new strategies, but rather in prompting implementation of known strategies. Thus, this example of changing behavior of appropriate antibiotic use was more about creating awareness of strategies than changing knowledge.

This study failed to demonstrate a "dose effect" in that those who had attended previous FMR (i.e., repeat attendees) were different (e.g., more receptive, more compliant) compared to responders who had attended only once. Because two exposures did not increase the rate of changing behavior, the dramatic effect of one exposure may be related to the importance of the topic and the potential seriousness of the outcome of overuse of antibiotic administration [21]. Based on our results, we concluded that only one exposure was needed to demonstrate an intended behavior change in practice.

We recognize that there are several limitations to this work. First, the surveys were conducted among primary care providers who chose to attend an intense week-long CME course. These practitioners were likely to be different from those who attend other CME activities or no CME at all. In addition, the response rate of implementing at least one strategy was moderate (66.3\%), leading to a likelihood of nonresponse bias. We also acknowledge that attendees selfreported a behavior change, but we do not know whether the changes were actually implemented in practice.

Overuse of antibiotic therapy is a common problem and has important public health implications. CME is an important medium to effect change in practice. Consistent with Forsetlund et al. [21], we found that a mixed interactive and didactic CME program was effective, especially given the seriousness of the outcome. By attending our CME sessions, learners developed awareness of the consequences of antibiotic overuse and were more likely to report implementing strategies for reducing antibiotic administration.

\section{Conflict of Interests}

The authors report no conflict of interests.

\section{Acknowledgment}

This work is submitted from the Office of Continuing Medical Education and Professional Development, University of Colorado School of Medicine, Aurora, Colorado.

\section{References}

[1] Accreditation Council for Continuing Medical Education, Accreditation Criteria, 2015, http://www.accme.org/requirements/accreditation-requirements-cme-providers/accreditationcriteria.

[2] B. S. Bloom, "Effects of continuing medical education on improving physician clinical care and patient health: a review of systematic reviews," International Journal of Technology Assessment in Health Care, vol. 21, no. 3, pp. 380-385, 2005.

[3] F. Goulet, E. Hudon, R. Gagnon, E. Gauvin, F. Lemire, and I. Arsenault, "Effects of continuing professional development on clinical performance: results of a study involving family practitioners in Quebec," Canadian Family Physician, vol. 59, no. 5, pp. 518-525, 2013.

[4] F. Légaré, A. Freitas, P. Thompson-Leduc et al., "The majority of accredited continuing professional development activities do not target clinical behavior change," Academic Medicine, vol. 90, no. 2, pp. 197-202, 2015.

[5] A. G. Mathew, R. Cissell, and S. Liamthong, "Antibiotic resistance in bacteria associated with food animals: a United States perspective of livestock production," Foodborne Pathogens and Disease, vol. 4, no. 2, pp. 115-133, 2007.

[6] N. Shehab, P. R. Patel, A. Srinivasan, and D. S. Budnitz, "Emergency department visits for antibiotic-associated adverse events," Clinical Infectious Diseases, vol. 47, no. 6, pp. 735-743, 2008.

[7] L. Sun, E. Y. Klein, and R. Laxminarayan, "Seasonality and temporal correlation between community antibiotic use and resistance in the United States," Clinical Infectious Diseases, vol. 55, no. 5, pp. 687-694, 2012.

[8] R. Gonzales, T. Anderer, C. E. McCulloch et al., "A cluster randomized trial of decision support strategies for reducing antibiotic use in acute bronchitis," JAMA Internal Medicine, vol. 173, no. 4, pp. 267-273, 2013.

[9] R. Gonzales, D. C. Malone, J. H. Maselli, and M. A. Sande, "Excessive antibiotic use for acute respiratory infections in the United States," Clinical Infectious Diseases, vol. 33, no. 6, pp. 757762, 2001.

[10] T. C. Jenkins, A. Irwin, L. Coombs et al., "Effects of clinical pathways for common outpatient infections on antibiotic prescribing," The American Journal of Medicine, vol. 126, no. 4, pp. 327.e12-335.e12, 2013.

[11] National Strategy for Combating Antibiotic-resistant Bacteria, http://www.whitehouse.gov/sites/default/files/docs/carb_national_strategy.pdf.

[12] R. S. Gibbs, C. Wieber, L. Myers, and T. Jenkins, "A continuing medical education campaign to improve use of antibiotics in primary care," Journal of Biomedical Education, vol. 2014, Article ID 537681, 6 pages, 2014.

[13] World Health Organization, "WHO's first global report on antibiotic resistance reveals serious, worldwide threat to public health," Tech. Rep., World Health Organization, 2014, http:// www.who.int/mediacentre/news/releases/2014/amr-report/en/.

[14] Centers for Disease Control. Get Smart, http://www.cdc.gov/ getsmart/.

[15] Centers for Disease Control, Threat Report 2013, http://www .cdc.gov/drugresistance/threat-report-2013/index.html.

[16] N. M. Clark, M. Gong, M. A. Schork et al., "Impact of education for physicians on patient outcomes," Pediatrics, vol. 101, no. 5, pp. 831-836, 1998. 
[17] M. S. Gerrity, S. A. Cole, A. J. Dietrich, and J. E. Barrett, "Improving the recognition and management of depression: is there a role for physician education?" Journal of Family Practice, vol. 48, no. 12, pp. 949-957, 1999.

[18] A. M. Gülmezoglu, A. Langer, G. Piaggio, P. Lumbiganon, J. Villar, and J. Grimshaw, "Cluster randomised trial of an active, multifaceted educational intervention based on the WHO Reproductive Health Library to improve obstetric practices," BJOG, vol. 114, no. 1, pp. 16-23, 2007.

[19] R. Laprise, R. Thivierge, G. Gosselin et al., "Improved cardiovascular prevention using best CME practices: a randomized trial," Journal of Continuing Education in the Health Professions, vol. 29, no. 1, pp. 16-31, 2009.

[20] F. Goulet, R. J. Gagnon, G. Desrosiers, A. Jacques, and A. Sindon, "Participation in CME activities," Can Fam Physician, vol. 44, pp. 541-548, 1998.

[21] L. Forsetlund, A. Bjørndal, A. Rashidian et al., "Continuing education meetings and workshops: effects on professional practice and health care outcomes," Cochrane Database of Systematic Reviews, no. 2, Article ID CD003030, 2009. 

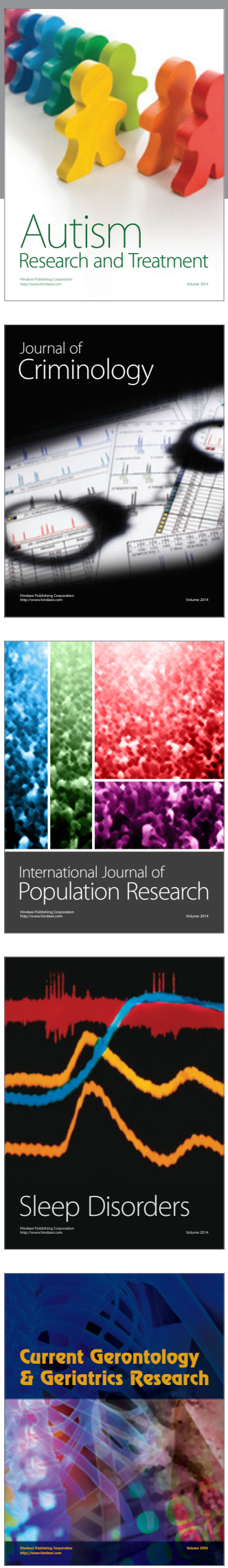
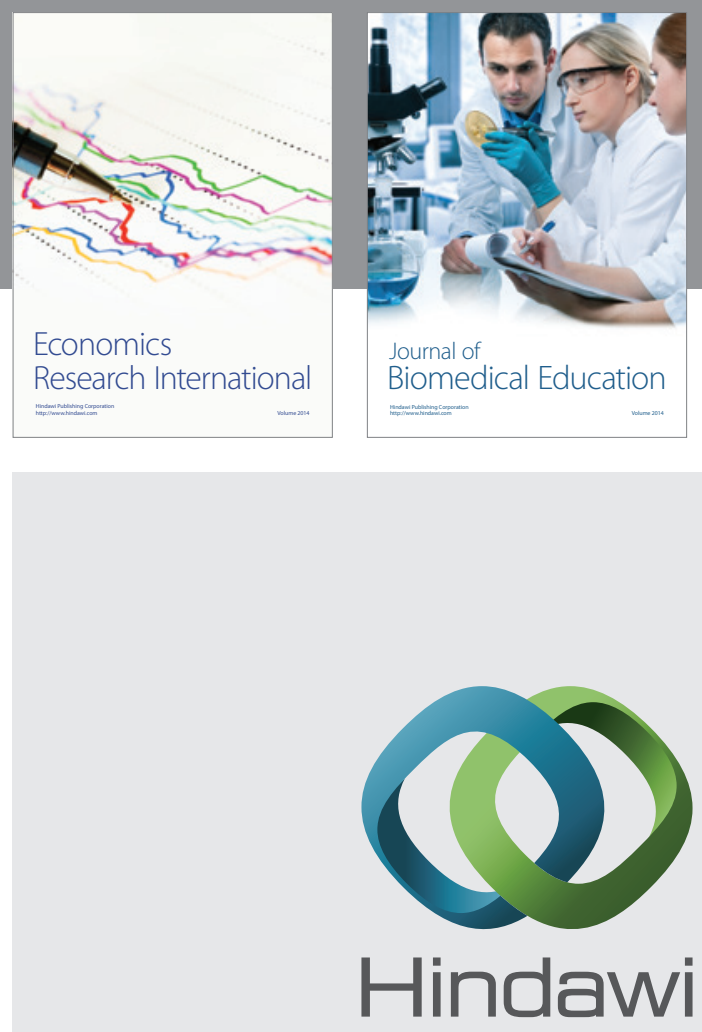

Submit your manuscripts at

http://www.hindawi.com
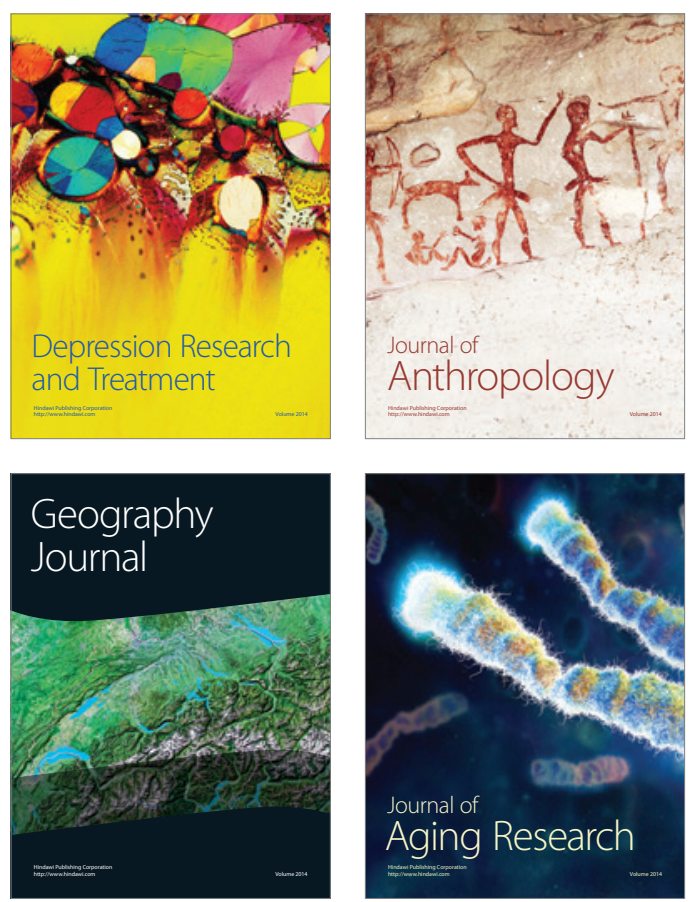
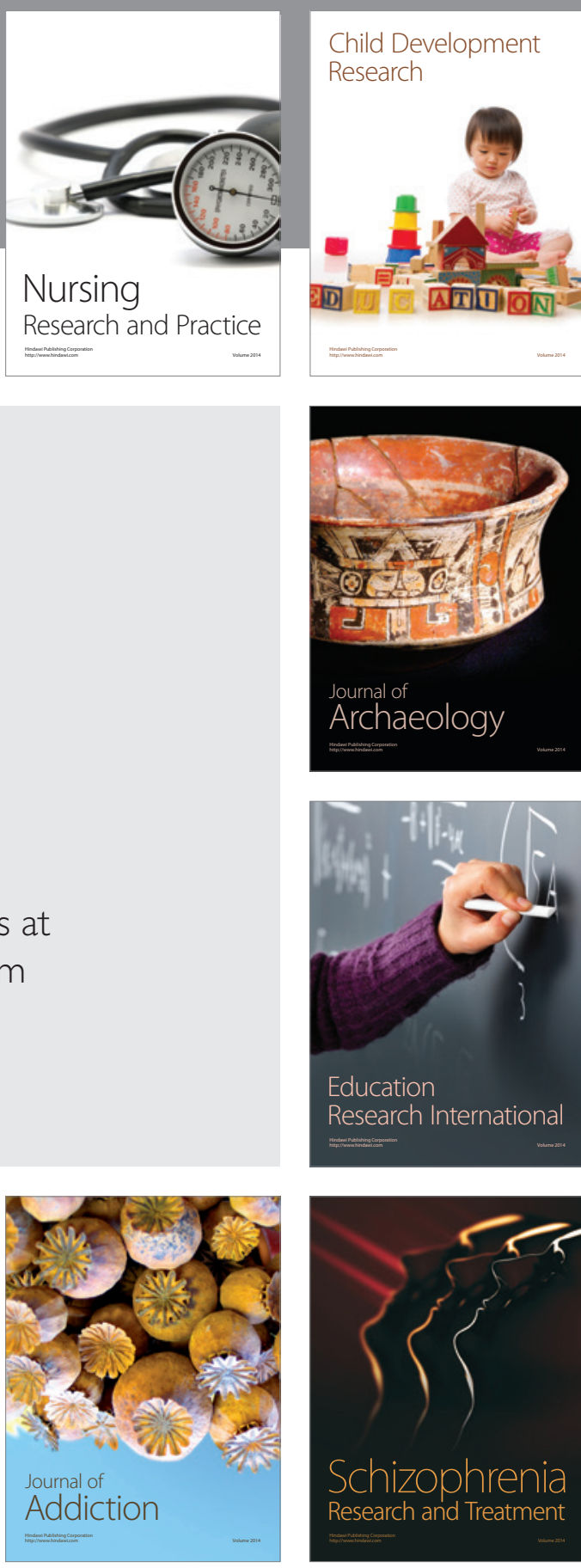

(D)
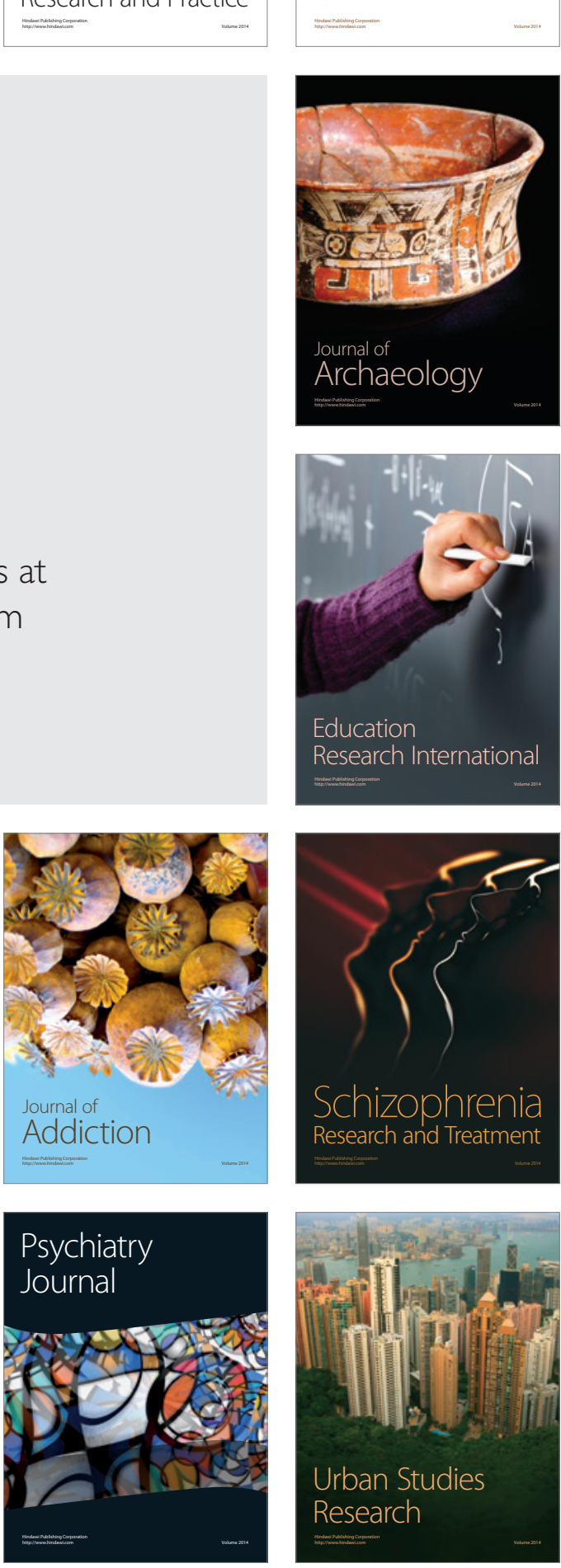